\title{
SOBRE EL GÉNERO ARMERIA EN LA PROVINCIA DE MÁLAGA (ANDALUCÍA, ESPAÑA)
}

\author{
Baltasar CABEZUDO*, Federico CASIMIRO-SORIGUER SOLANAS \\ y Andrés V. PÉREZ LATORRE \\ Departamento de Biología Vegetal. Facultad de Ciencias. Universidad de Málaga. \\ Campus de Teatinos. 29071, Málaga \\ *Autor para correspondencia: bcabezudo@uma.es
}

Recibido el 1 de septiembre de 2015, aceptado para su publicación el 3 de octubre de 2015

\begin{abstract}
RESUMEN. Sobre el género Armeria en la probincia de Málaga (Andalucía, España). Las poblaciones del genero Armeria localizadas en las sierras de Aguas y Alcaparaín, ambas en la provincia de Málaga, se han incluido tradicionalmente en Armeria villosa subsp. carratracensis (Bernis) Nieto Feliner. El estudio del material de ambas sierras ha puesto de manifiesto que en realidad se trata de dos entidades taxonómicas distintas. Las poblaciones de la Sierra de Alcaparaín, sobre dolomías, se identifican como Armeria grajoana Casimiro-Soriguer Solanas \& Cabezudo, sp. nov. Las poblaciones de Sierra de Aguas, sobre serpentinas/ peridotitas, se identifican como Armeria villosa subsp. serpentinicola Cabezudo, Pérez Latorre \& CasimiroSoriguer Solanas, subsp. nov.
\end{abstract}

Palabras clave. Sierra de Aguas, Sierra de Alcaparaín, Armeria grajoana, Armeria villosa subsp. sepentinicola

SUMMARY. Contribution to genus Armeria in Malaga province (Andalusia, Spain). The populations of the genus Armeria located in the mountain ranges of Aguas and Alcaparain, both in the province of Malaga (Spain), have traditionally been included in Armeria villosa subsp. carratracensis (Bernis) Nieto Feliner. The studied material, collected in both ranges, has shown that two independent taxa exist. The populations of the Sierra de Alcaparaín on dolomites, were identified as Armeria grajoana Casimiro-Soriguer Solanas \& Cabezudo, sp. nov. The populations of Sierra de Aguas, living on peridotites, were identified as Armeria villosa subsp. sepentinicola Cabezudo, Perez Latorre \& Casimiro-Soriguer Solanas, subsp. nov.

Key words. Sierra de Aguas, Sierra de Alcaparaín, Armeria grajoana, Armeria villosa subsp. sepentinicola

\section{INTRODUCCIÓN}

En Mayo del 2014 y en el marco de un proyecto sobre el estudio de la flora y vegetación de Sierra de Alcaparaín (Málaga; Casarabonela/ Ardales/ Carratraca) localizamos una pequeña población de una especie del género Armeria que nunca antes habíamos visto en el marco biogeográfico del sector Rondeño (provincia Bética) (Pérez Latorre \& Cabezudo, 2002). La población se localizaba en las crestas de la sierra, en grietas de rocas dolomíticas, 
muy castigadas por el viento y por la presión del ganado. La mayoría de los individuos localizados se encontraban ramoneados y solo unos pocos habían llegado a florecer.

Para la identificación del material utilizamos, sin resultado, la excelente monografía sobre el género Armeria (Nieto Feliner, 1990) y en ella localizamos una referencia a la Sierra de Alcaparaín (Sierra de Caparaín (sic), pr. Carratraca, 19 jun. 1930, leg. C. Vicioso) como indicación locotípica (in. loc.) de A. villosa subsp. carratracensis (Bernis) Nieto Feliner in Anales Jard. Bot. Madrid 44: 337(1987). Como basiónimo de esta subespecie se indica a A. maritima subvar. carratracensis Bernis in Anales Inst. Bot. Cavanilles 12 (2): 122 (1955) y en la iconografía de la misma se representa un ejemplar recolectado en la Sierra de Aguas, pr. Carratraca, Málaga (MA 360159) (Nieto Feliner, 1990: 687).

El estudio del material recolectado en la Sierra de Alcaparaín dio como resultado diferencias significativas con A. bourgaei (s.l.), A. filicaulis (s.l.), A. malacitana, A. colorata y A. villosa (s.1.). Todo el material visto, en pliegos y en el campo, de A. malacitana, $A$. colorata y A. villosa (s.l.) presentan siempre la corola blanca. A. bourgaei, A. filicaulis y A. malacitana viven siempre sobre calizas, dolomías y micaesquistos. A. villosa subsp. carratracensis (sensu Nieto Feliner, 1987) y A. colorata lo hacen siempre sobre serpentinas, ambas con corola blanca (Nieto Feliner, 1987; Pérez Latorre et al., 2013).

Dado que el único material previo localizado de este género en la Sierra de Alcaparaín era el recolectado por Vicioso en 1930 (Nieto Feliner, 1990), solicitamos este pliego (MA 145420) al Real Jardín Botánico de Madrid (MA), al ser dicho material el Holotypus (fig. 1) de A. villosa subsp. carratracensis (sensu Nieto Feliner, 1987), nos fue remitido solo una copia escaneada. El pliego (MA 145420) consta de varios ejemplares y 4 etiquetas, una primera manuscrita (a tinta) con el nombre de Armeria villosa Gir. var. carratracensis C. Vic. (nov. var.). In dumetis. Sierra Caparain. Carratraca (Málaga). 19.VI. 1930. C. Vicioso. Corollis rosas (margen inferior y a lápiz). Una segunda etiqueta (a máquina) que reproduce la etiqueta anterior excepto el termino referido al color de la corola. Una tercera (a maquina y manuscrita) con el nombre de Armeria maritima (Mill.) Willd., sensu nov (tachado), subsp. eumaritima sensu Bern., var. villosa (Gir) Bern. (series A), subvar. carratracensis Bern. det. Bernis. La cuarta etiqueta corresponde a la revisión que para Flora Iberica realizó el Dr. G. Nieto Feliner y dice lo siguiente: A. villosa Girad subsp. carratracensis (Bernis) Nieto Feliner. Holotypus (A. maritima subvr. carratracensis Bernis). Anales Inst. Bot. Cavanilles 12 (2):122 y Lam 36 A (1955). Gonzalo Nieto Feliner. Real Jardín Botánico. Madrid. 1986 (existe una flecha que índica como holotypus al ejemplar central del pliego).

Armeria maritima subvar. carratracensis fue descrita como subvar. nova por Bernis (Bernis 1955:122), utilizando para su confusa descripción morfológica material de dos entidades distintas, el pliego anteriormente descrito (MA 145420) y dos pliegos procedentes de Sierra Llana de Antequera (MA145419) y Alfarnate (MA 145441) que en realidad corresponden a A. villosa subsp. longiaristata como veremos posteriormente. Como tipo indica el ejemplar fotografiado en la lámina 36 (Bernis 1955: 234) del pliego recolectado por Vicioso (MA 145420), que corresponde al ejemplar (fotografía invertida) reseñado por Nieto Feliner en el pliego en cuestión. Al comparar la descripción de Bernis (Bernis 1955:122) de esta subvar. carratracensis con la de A. villosa subsp. carratracensis (NietoFeliner, 1990: 689) comprobamos que tenían que haber sido realizadas sobre materiales distintos.

Para los datos ecológicos y morfológicos de Armeria maritima subvar. carratracensis, Bernis (1955:124-125) tiene en cuenta tres 
pliegos correspondientes a las localidades de Sierra de Caparaín (calizas y dolomías), Sierra Llana de Antequera (calizas), recolectados ambos por Vicioso en 1930, y un pliego recolectado en la localidad de Alfarnate (calizas) por Gros en 1919. Es de suponer que Bernis no conoce ni visita el lugar de recolección de estos pliegos, por lo que, utilizando un desconocido mapa geológico, indica que "por lo menos dos de las tres localidades conocidas corresponden a regiones donde dominan pizarras silúricas o esquistos arcaicos, acompañados de rocas peridotíticas”. Hemos estudiado el material de Sierra Llana de Antequera y de Alfarnate mencionados por Bernis (1.c.) e identificados por él como A. maritima subvar. carratracensis Bern. (MA145419) y A. maritima subvar. genuina Bern. (MA 145441) y que posteriormente fueron ambos identificados en 1987 por Nieto Feliner como A. villosa subsp. longiaristata (Boiss. \& Reuter) Nieto Feliner. La utilización por Bernis del material procedente de Sierra Llana de Antequera, que el mismo considera que no pertenece claramente a la subvar. carratracensis, para la descripción de esta subvar., ha motivado que en dicha descripción aparezcan algunos datos desviantes respecto al material de Sierra de Alcaparaín. Tanto en Sierra Llana como en las sierras de Alfarnate no se localiza ningún afloramiento de peridotitas ni de pizarras, mientras que la Sierra de Alcaparaín (Caparaín) es, casi en su totalidad, de naturaleza dolomítica, con una pequeña mancha de peridotitas en su contacto con la Sierra de Aguas, esta última íntegramente de naturaleza peridotítica (López González, 1975).

Quizás esta consideración edafológica de Bernis asignando un sustrato peridotítico a este material de la Sierra de Alcaparaín y considerar que, geológicamente, todo el termino municipal de Carratraca es de peridotitas, es lo que ha podido generar el conflicto de incluir bajo un mismo epíteto especifico, carratracensis, a dos entidades distintas del género Armeria que viven en el mismo termino municipal (Carratraca) pero sobre sustratos geológicos muy diferentes: Sierra de Alcaparín (dolomías) y Sierra de Aguas (peridotitas y serpentinas). El propio Nieto Feliner (1987: 337) al publicar la combinación nueva de $A$. villosa subsp. carratracensis ignora la localidad del pliego de Alcaparaín y asigna como distribución de la subespecie Sierra de Aguas (Carratraca) y Sierra Parda (Tolox) ambas sierras totalmente constituidas por peridotitas.

Para ver realmente sobre que sustrato geológico crece el material de Armeria villosa var. carratracensis recolectado por Vicioso en la Sierra de Caparaín, hemos acudido al trabajo sobre la Vegetación y Flora Forestal de la Provincia de Málaga (Ceballos y Vicioso, 1933: 83 y 149) en el que dice que “ En la vertiente oriental de la Sierra de Caparaín aparece un suelo con reacción básica, pobre, de poco fondo y con frecuentes afloramientos de la roca madre (calizas triásicas)”. Por tanto consideramos que el material de Armeria villosa var. carratracensis recolectado por Vicioso lo fue sobre sustratos dolomíticos y nunca sobre peridotitas (serpentinas), así lo atestigua además la presencia de numerosas especies calcícolas-dolomitícolas en la Sierra de Caparaín (Ceballos y Vicioso, 1933: 84). En un estudio de la Sierra de Aguas (López González, 1975) se cita Armeria allioides Boiss. en las peridotitas de la parte alta de la sierra. No hemos visto este material, pero todo lo recolectado por nosotros en esta sierra corresponde a A. villosa subsp. carratracensis (sensu Nieto Feliner, 1987).

Sin justificación morfológica, ni fitosociológica, ni ecológica, Rivas Martínez (1988:115) publica Armeria carratracensis (Bernis) Rivas Martínez comb. nov. e indica como basiónimo a Armeria maritima subvar. carratracensis Bernis in Anales Inst. Bot. Cavanilles 12 (2):122 (1955). Suponemos que Rivas Martínez no ha visto el tipo (MA 145420) pues no hace referencia al mismo en 
su publicación, y en el pliego de referencia (MA 145420) no aparece ninguna información manuscrita sobre su utilización por el Dr. S. Rivas Martínez que haga referencia a esta combinación, es por tanto, como muchas, una combinación teórica y sin fundamentos. Lo indudable es que Rivas Martínez se está refiriendo a las poblaciones sobre peridotitas tal como se indica en Rivas Martínez et al. (1991: 16) donde dice claramente que la especie es: "Bermejense. En tomillares sobre litosuelos serpentinizados” y en otras dos publicaciones posteriores (Rivas Martínez 2011a: 326 y 2011b: 498) donde se ratifica en su criterio de que es una entidad característica de comunidades sobre peridotitas (Asperulo asperrimae-Staehelinetum baeticae Rivas Goday \& Rivas martinez 1969). Nieto Feliner (1990: 855) sinonimiza esta combinación a Armeria villosa subsp carratracensis y Rivas Martínez et al. (1991:16) sinonimizan la subsp carratracensis de Nieto Feliner a su A. carratracensis. Consideramos por tanto que esta combinación de Rivas Martínez (1988) no es válida al considerar como basiónimo del material sobre peridotitas a lo descrito por Bernis (1955). Rivas Martínez nunca hace mención al pliego de referencia (holotypus) recolectado por Vicioso en 1930 ni al hábitat (dolomías) de los materiales empleados por Bernis para Armeria maritima subvar. carratracensis (Bernis, 1955) y por tanto consideramos que el epíteto carratracensis no puede aplicarse a las plantas localizadas sobre peridotitas/serpentinas.

La rigidez del Código Internacional de Nomenclatura Botánica complica, en determinados casos como este, la delimitación y denominación clara de algunas entidades taxonómicas, siendo por el contrario muy laxo con las condiciones necesarias para dar por válida algunas nuevas combinaciones en las que los autores de las mismas ni siquiera han manejado o visto material de los taxones sobre las que las realizan. Empezar de cero con este complicado grupo de especies y olvidarse de la pesada e histórica mochila taxonómica y nomenclatural podría aclarar mucho la diversidad del género Armeria.

C. Vicioso nunca publicó su variedad Armeria villosa var. carratracensis tal como aparece en el pliego MA 145420. El epíteto carratracensis, en el sentido de C. Vicioso, se refiere al termino municipal de Carratraca ya que en su zona mas occidental se localiza parte de la Sierra de Alcaparaín y no a la Sierra de Carratraca (llamada también de Aguas) que se localiza casi en su totalidad en los términos de Casarabonela y Alora, con una porción muy pequeña en el oriente del término de Carratraca. Todo lo publicado bajo los nombres de $A$. villosa subsp. carratracensis (Bernis) sensu Nieto Feliner y A. carratracensis sensu Rivas Martínez corresponden a materiales sobre serpentinas y nunca hacen referencia a sustratos calizos o dolomíticos tal como indica C. Vicioso para su material de la var. carratracensis. Con el fin de diferenciar claramente las poblaciones sobre serpentinas de las que viven sobre sustratos dolomíticos y no utilizar para ambas entidades el confuso epíteto de carratracensis es por lo que proponemos el epíteto de grajoana (en referencia al pico de El Grajo) para las poblaciones sobre dolomías reseñadas por $\mathrm{C}$. Vicioso, y proponer una subespecie nueva para las poblaciones sobre serpentinas. Por todo lo anterior consideramos que, a pesar de compartir hasta el momento el mismo holotipo, (Sierra de Alcaparaín, MA 145420), A. maritima subvar. carratracensis Bernis (calizas y dolomías) y A. villosa subsp. carratracensis (Bernis) Nieto Feliner (peridotitas/serpentinas), son dos entidades taxonómicas distintas, y en consecuencia proponemos lo siguiente:

Armeria grajoana Casimiro-Soriguer Solanas \& Cabezudo, sp. nov.

Armeria maritima subvar. carratracensis Bernis in Anales Inst. Bot. Cavanilles 12 (2) : 


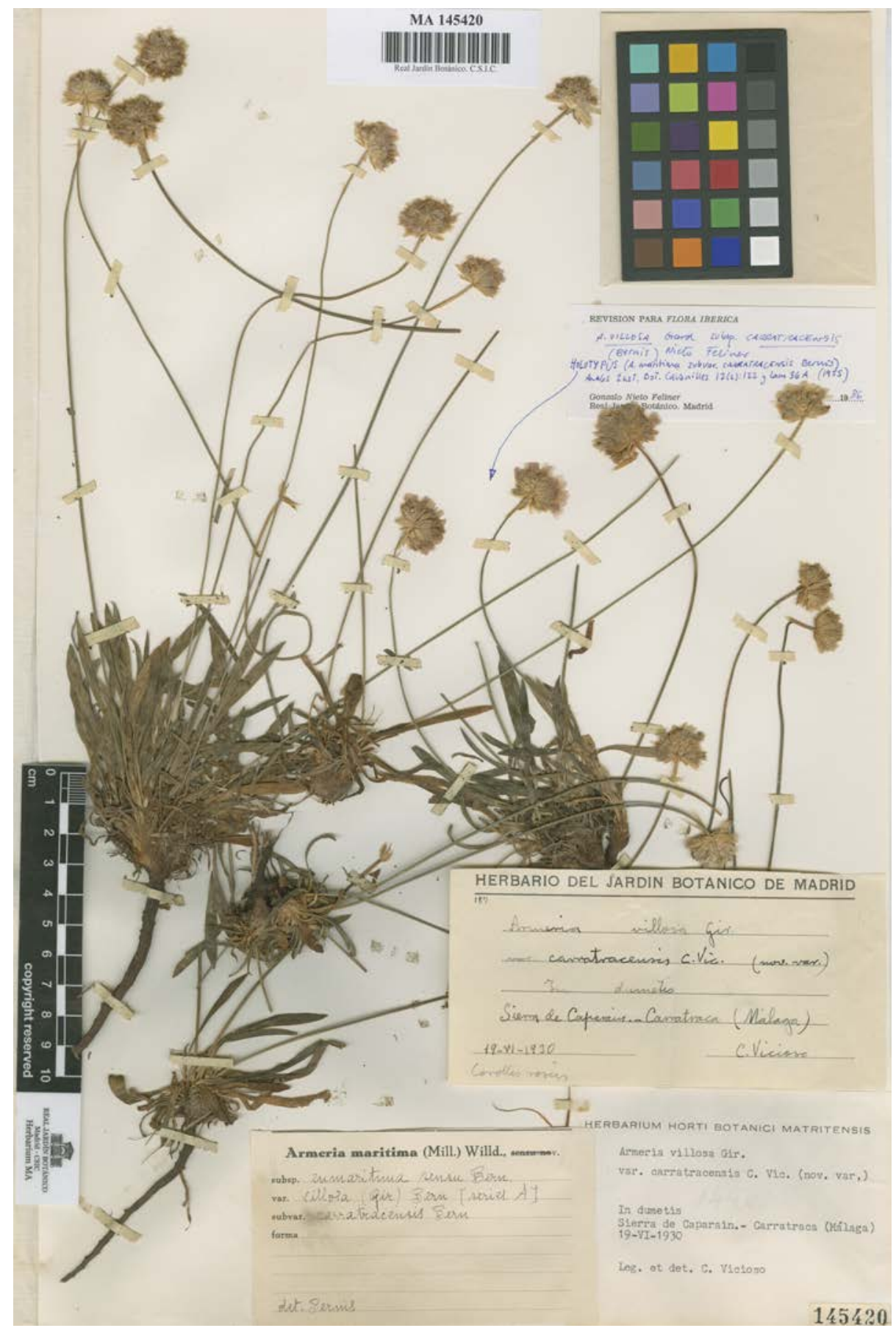

Figura 1. Holotypus de Armeria grajoana. Holotype of Armeria grajoana. 
122 (1955) (basión. solo el typus).

A. villosa var. carratracensis C. Vicioso nom. in sched. (MA 145420).

Holotypus: Sierra de Caparaín. Carratraca (Málaga), 19.VI.1930, leg. C. Vicioso (MA 145420) (sub. A. villosa var. carratracensis C. Vicioso) (fig. 1)

Hemicriptófíto con cepa ramificada y vertical de ramas muy apretadas. Cepa cubierta de hojas viejas que se deshacen en fibras. Planta glabra, excepto generalmente las hojas. Hojas (25)30-70 (95)x(2,5)3-5(6) mm., homomorfas, lanceoladas, patentes o erectas, generalmente atenuadas en un largo peciolo, con margen escarioso estrecho, de glabras a generalmente ciliadas exclusivamente en el margen; con granulaciones blancas en el limbo. Escapos (12)15-18(20) cm., de rectos a flexuosos en su mitad superior, glabros. Involucro 1,4-1,8 (2) $\mathrm{cm}$. Vaina involucral de longitud similar al diámetro del involucro o frecuentemente algo menor 4/5. Brácteas involucrales 7-12, lisas, glabras, con margen escarioso, generalmente crecientes en tamaño de las externas a las

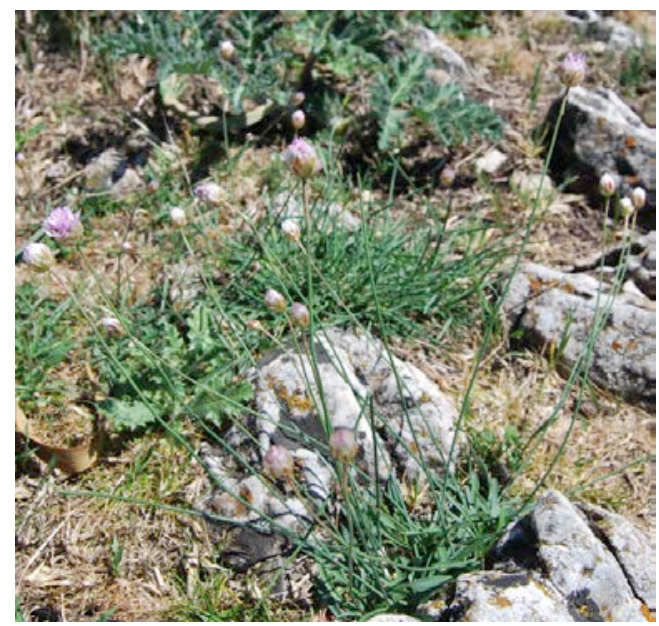

Figura 2. Armeria grajoana. Aspecto general de la planta. Biotype . internas; las mas externas gradualmente atenuadas hacia el ápice; las medias e internas ovadas contraídas en el ápice y mucronadas, las internas con amplio margen escarioso, con el mucrón y la parte central frecuentemente rojizas. Brácteas espiculares totalmente escariosas y concoloras de tamaño similar a las brácteas involucrales internas, ovadas. Cáliz 6,5-7 (8) mm., holopleurótico, con hileras de pelos densas, pelos largos que prácticamente ocultan los surcos intercostales; espolón 0,4-0,5 mm., de 1/5 a 1/6 del tamaño del tubo. Lóbulos 1,5-2 mm., marcados, triangulares, cortamente aristados; lóbulos 1/5 de la longitud del cáliz. Corola rosa-púrpura. (figs. 2 y 3 )

Rootstock branched. Scapes up to $18 \mathrm{~cm}$. Involucre up to $2 \mathrm{~cm}$. Involucral bracts scarcely thick, with increasing size from the external towards the internal ones. Bracts spicular and totally scarious, with similar size to the involucral internal ones. Calyx up to $7 \mathrm{~mm}$., shortly-awned. Corolla purplish-pink.

Fenología. Floración: IV-VI. Fructificación: V-VII

Ecología. Rupícola, subrupícola y matorrales xerófilos sobre suelos dolomíticos.

Distribución. Endemismo de la Sierra de Alcaparaín (Málaga)

Biogeografía. Subsector Rondense (sector Rondeño, provincia Bética, Subregión Mediterránea-Occidental).

Altitud: $1000-1293 \mathrm{~m}$

Fitosociología. Comunidades dolomitícolas del Lavandulo lanatae-Ulicetum baetici y Galio baetici-Thymetum granatensis Se acompaña de especies como: Thymus granatensis subsp. granatensis, Sideritis incana subsp. occidentalis, Ulex baeticus subsp. baeticus, Stipa tenacissima, Arenaria armerina, Jurinea pinnata o Ptilostemon hispanicus.

Categoría de amenaza (UICN). En Peligro Crítico (CR) (B1ab(ii,iv);C2a(i)). Se propone su inclusión en el Listado Andaluz de 


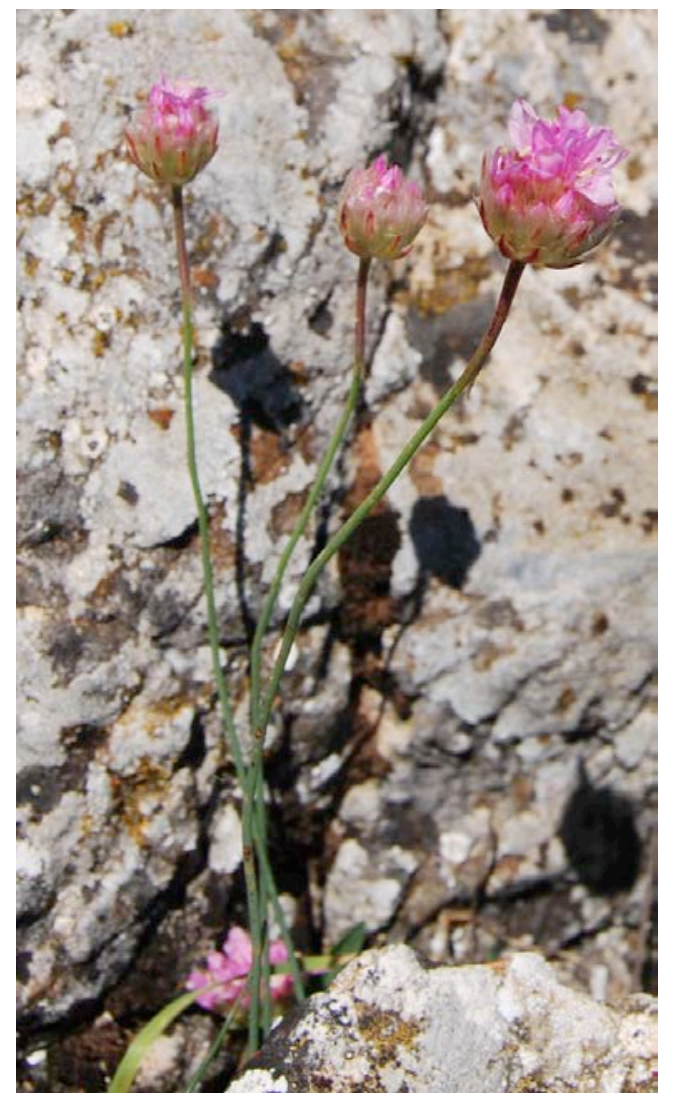

Figura 3. Armeria grajoana. Escapo e inflorescencia. Inflorescence branch.

Especies Silvestres en Régimen de Protección Especial incluido el Catálogo Andaluz de Especies Amenazadas como especie amenazada con la categoría de En Peligro de Extinción (EN).

Material estudiado: Málaga: Sierra de Caparaín (Cádiz?). 19/07/1930?. Vicioso (EMMA 11564). Carratraca. Sierra de Caparain. In dumetis. 19/06/1930. Vicioso (MA 145420) Holotypus; ibídem. Sierra de Alcaparaín. El Grajo. Espolón norte. UF3577. Rupícola. Paredes verticales y repisas. Dolomías.1150-1280 m.s.m. 05/06/2014. Casimiro-Soriguer Solanas (MGC 79121); ibídem. Blanquizar dolomítico en laderas pedregosas de fuertes pendientes. Dolomías disgregadas.
05/06/2014. Casimiro-Soriguer Solanas (MGC 79131); ibídem. Cabecera del arroyo del Conejo, ramal sureste. UF3478. Zona rocosa. Rupícolas y subrupícolas. Dolomías. 29/05/2015. Cabezudo, Pérez Latorre, Hidalgo \& Casimiro-Soriguer Solanas (MGC 81159). Carratraca/Casarabonela. Sierra de Alcaparaín. Crestería entre el Pico Valdivia y El Grajo. UF 3577. Zona muy rocosa. Dolomías. 1160-1293 m. s. m. 2/05/2014. Cabezudo, CasimiroSoriguer Solanas \& García Sánchez (MGC 78499). Casarabonela. Sierra de Alcaparaín. Cerro de la Canana. UF3379. Roquedos. Dolomías. 1100-1182 m.s.m. 08/05/2014. Cabezudo \& Casimiro-Soriguer Solanas (MGC 78573); ibídem. 23/05/2014. Cabezudo, Pérez Latorre \& Casimiro-Soriguer Solanas (MGC 78856); ibídem. 29/05/2015. Cabezudo, Pérez Latorre, Hidalgo \& CasimiroSoriguer Solanas (MGC 81150); ibídem. Pico del Grajo y alrededores. UF 3577. Crestería rocosa. Dolomías. 23/05/2014. Cabezudo, Pérez Latorre \& Casimiro-Soriguer Solanas (MGC 78846, 78842).

Observaciones. Hemos revisado el material del genero Armeria utilizado para la realización de las Floras Vasculares de Andalucía Occidental (Valdés et al., 1987) y de Andalucía Oriental (Blanca et al., 2009) con el fin de localizar algún material semejante, en morfología y ecología, al recolectado por nosotros y C. Vicioso en la Sierra de Alcaparaín. En el Herbario de la Universidad de Sevilla (SEV) hemos localizado un pliego (original y tres duplicados) con material parecido al de Alcaparaín recolectado en Sierra Horconera: Pico Bermejo (Córdoba) (SEV 82315, 82316, 82314 y 76396) identificados originalmente como A. maritima subsp. alpina (Willd.) Pinto da Silva y publicado como tal por Devesa y Pinto da Silva (1984) y en Valdés et al. (1987: 297). Los pliegos fueron revisados para Flora iberica y tienen una etiqueta de corrección donde dice: "Puede ser A.villosa x A. bourgaei (no es A. alpina). Gonzalo Nieto Feliner. Real Jardín Botánico. Madrid . X. 1987 ”. Este material, procedente de Sierra Horconera, ha sido descrito posteriormente como Armeria trianoi (Nieto Feliner et al., 2001:75). Visto el 
material de esta especie hemos considerado que, a pesar del aparente parecido morfológico y a la similitud ecológica (calizas y dolomías), no se corresponde claramente con el recolectado por nosotros en Sierra de Alcaparaín, motivo por el cual, y hasta nuevos estudios, mantenemos independientes las identidades especificas de las poblaciones de Alcaparaín y Horconera.

Las diferencias mas significativas de esta especie con A. colorata, A. villosa subsp. serpentinicola, $A$. villosa subsp. longiaristata y A. trianoi están representadas en la tabla 1.

\section{Armeria villosa subsp. serpentinicola}

Cabezudo, Pérez Latorre \& CasimiroSoriguer Solanas, subsp. nov.

- Armeria villosa subsp. carratracensis (Bernis) sensu Nieto Feliner in Anales Jard. Bot. Madrid 44:337(1987), excl. typus; in Flora Iberica 2: 689 (1987), excl. typus.

- A. carratracensis (Bernis) sensu Rivas-Martinez in Lagascalia 15(extra) :115 (1988), excl. typus.

- A. allioides sensu López in Acta Botanica Malacitana 1: 120 (1975), non Boiss., Voy. Bot. Espagne 2: 525 (1841), nom. illeg.

Holotypus: Málaga: Carratraca. Sierra de Aguas. UF38. Peridotitas. 2/06/2004. B. Cabezudo \& O. Gavira. (MGC 63048) (fig. 4).

Hemicriptófito, con cepa vertical no ramificada. Cepa cubierta de hojas viejas que se deshacen en fibras. Hojas 30- 90 (110) x 1,5-7 mm. homomorfas, numerosas, de linear-lanceoladas a lanceoladas, trinervadas, glabras o ciliadas en el margen, este, a veces sinuado, limbo con granulaciones blancas, las externas generalmente rojizas. Escapos 25-45 cm., rectos, glabros. Involucro 17-23 $\mathrm{mm}$. Vaina involucral $16-34 \mathrm{~mm}$., de longitud similar o superior al diámetro del involucro. Brácteas involucrales 13-20, lisas ( las externas a veces rugosas), consistentes, las externas

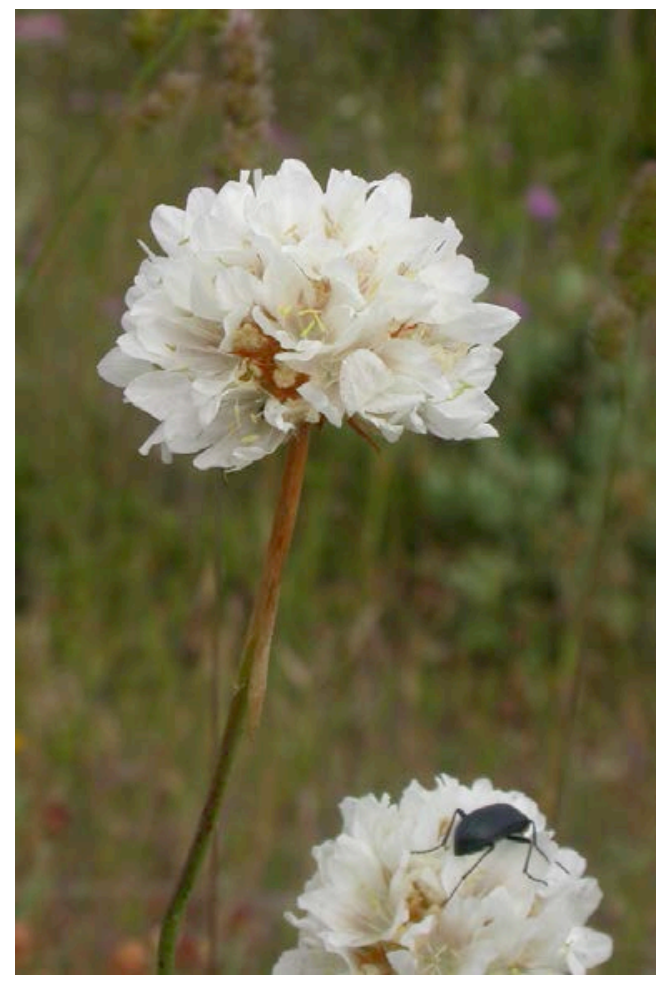

Figura 5. Armeria villosa subsp. serpentinicola. Escapo e inflorescencia. Inflorescence branch.

de mucronadas a largamente cuspidadas, de tamaño decreciente hacia el interior. Brácteas espiculares no enteramente escariosas, de iguales a algo más largas que las involucrales internas. Cáliz 8-9,5 mm., holopleurótico, con hileras de pelos densas; espolón 0,3-1,3 mm, de $1 / 4$ a $3 / 5$ de la longitud del tubo; lóbulos 2-3 mm., triangulares muy marcados y largamente aristados, de $1 / 5$ a 1/3 de la longitud del cáliz. Corola blanca. (figs.5 y 6).

Rootstock vertical and unbranched. Numerous leaves, the external reddish. Scapes up to $45 \mathrm{~cm}$. Involucre up to $23 \mathrm{~mm}$. Involucral bracts with decreasing size towards the inside. Involucral bracts spicular, not entirely scarious, equal to slightly longer than the internal ones. Calyx up to $9.5 \mathrm{~mm}$., long-awned. Corolla white. 


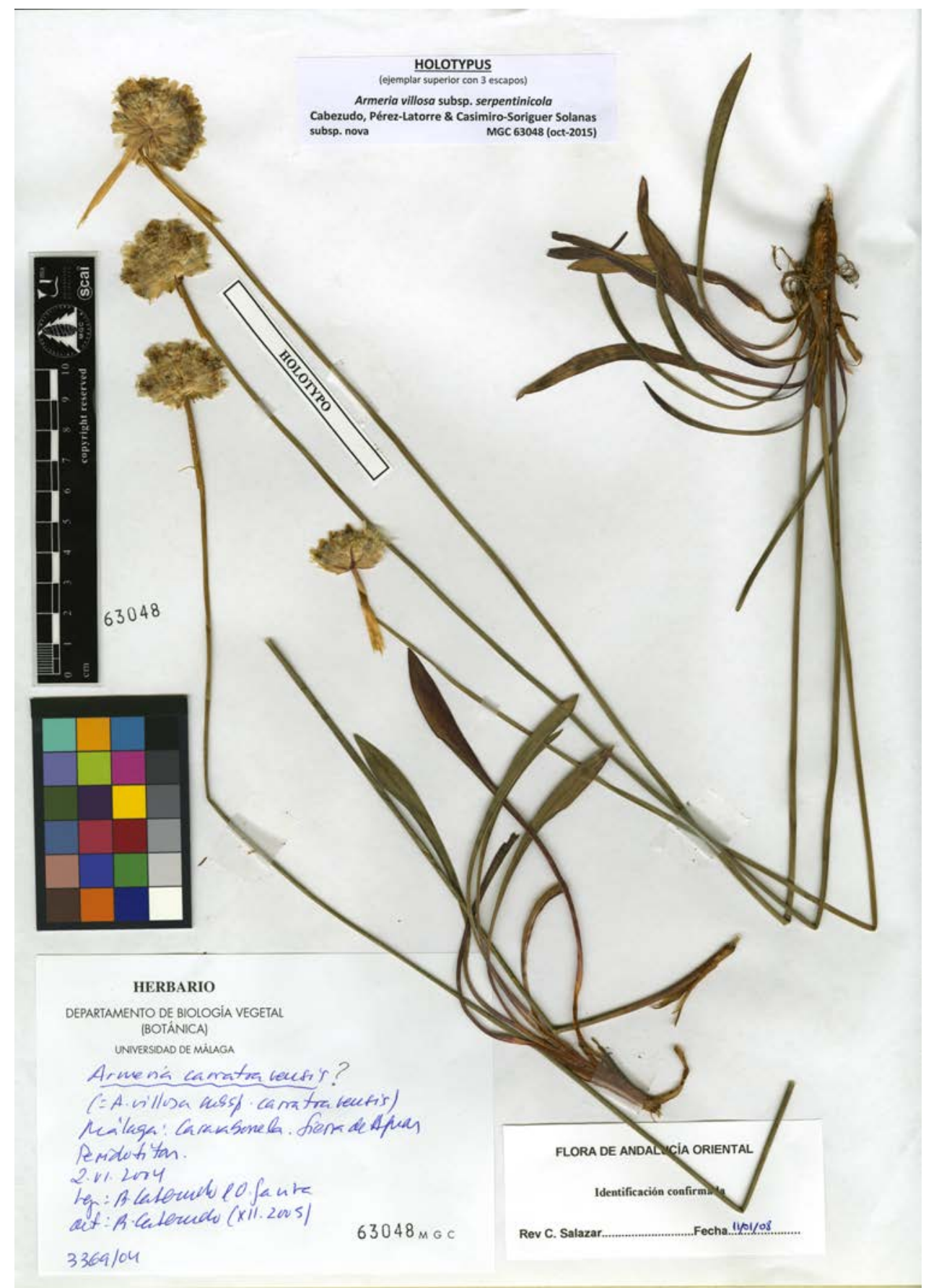

Figura 4. Holotypus de Armeria villosa subsp. serpentinicola. Holotype of Armeria villosa subsp. serpentinicola. 
Fenología. Floración: IV-VI. Fructificación: V-VII

Ecología. Rupícola y subrupícola sobre suelos peridotíticos.

Distribución. Endemismo serpentinófito de la Sierra de Aguas (Málaga).

Biogeografía. Subsector Carratracense (sector Bermejense, provincia Bética, Subregión Mediterránea-Occidental).

Altitud. 750- $949 \mathrm{~m}$

Fitosociología. Comunidad serpentinícola del Galio boissierani-Staehelinetum baeticae. Se acompaña de especies como: Cerastium boissieri, Sesamoides canescens, Centaurea carratracensis, Alyssum serpyllifolium subsp. malacitanum, Galium boissieranum y Ptilotrichum longicaule.

Categoría de amenaza (UICN). En Peligro Crítico (CR) (B1ab(ii,iv);C2a(i). Se propone su inclusión en el Listado Andaluz de Especies Silvestres en Régimen de Protección Especial incluido el Catálogo Andaluz de Especies Amenazadas como especie amenazada con la categoría de En Peligro de Extinción (EN).

Material estudiado. Málaga: Carratraca. Sierra de Aguas. Entre Álora y Carratraca. UF38. Serpentinas. 800 m.s.m. 14/06/1973. Talavera \& Valdés. (MGC 12652); ibídem. 13/05/1993. Asensi \& Díez. (MGC 37088); ibídem. UF38. Peridotitas. 2/06/2004. B. Cabezudo \& O. Gavira. (MGC 63048).

Observaciones. En un interesante trabajo sobre hibridaciones en el género Armeria y mas concretamente sobre el origen híbrido de A. villosa subsp. carratracensis (Nieto Feliner et al., 1996) se pone de manifiesto la relación de esta subespecie con $A$. colorata (peridotitas) y A. villosa subsp. longiaristata (calizas/dolomias). El material utilizado de $A$. villosa subsp carratracensis (Bernis) Nieto Feliner para este estudio procede de la Sierra de Aguas (peridotitas/serpentinas), por lo que los resultados no pueden ser aplicados a la entidad A. grajoana de la Sierra de Alcaparaín

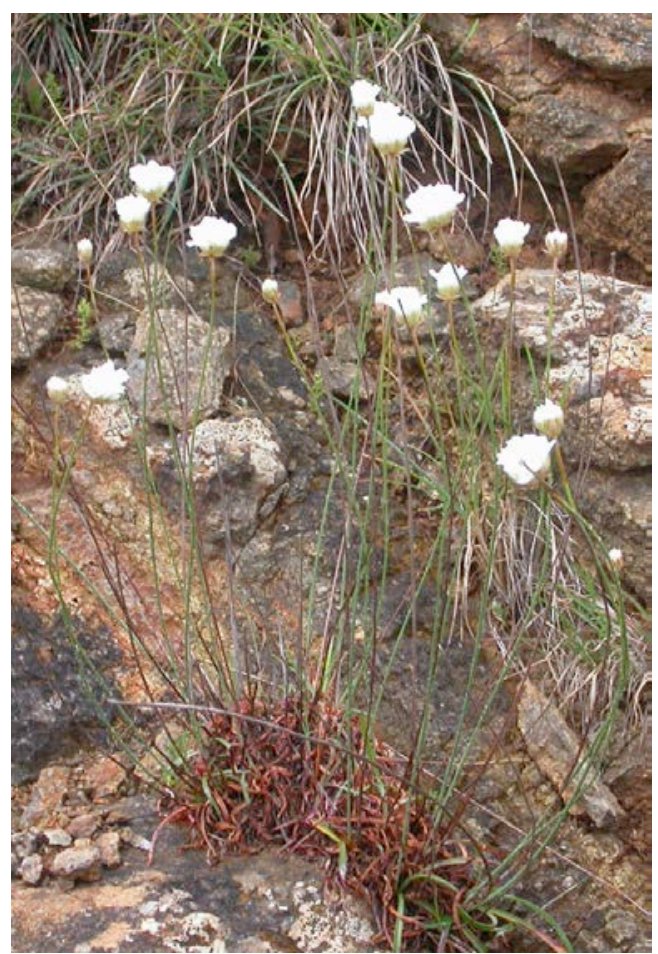

Figura 6. Armeria villosa subsp. serpentinicola. Aspecto general de la planta. Biotype.

(dolomías) y sí a la nueva entidad A. villosa subsp. sepentinicola.

Nuestras observaciones de campo y la revisión de material de herbario de Armeria recolectado sobre serpentinas nos hacen apoyar las estrechas relaciones que esta subespecie tiene con A. colorata (Nieto Feliner et al., 1996). Por el momento, y a la espera de un estudio mas completo de todo el grupo, consideramos que A. villosa subsp. serpentinicola es endémica de la Sierra de Aguas y que junto con A. colorata forman una entidad taxonómica diferenciada del conjunto de A. villosa s.l.

En dos trabajos realizados para evaluar el grado de amenaza de especies de la flora silvestre española (Cabezudo et al. 2000: 55 y 2003: 604) los datos relativos a Armeria villosa subsp carratracensis sensu Nieto Feliner 


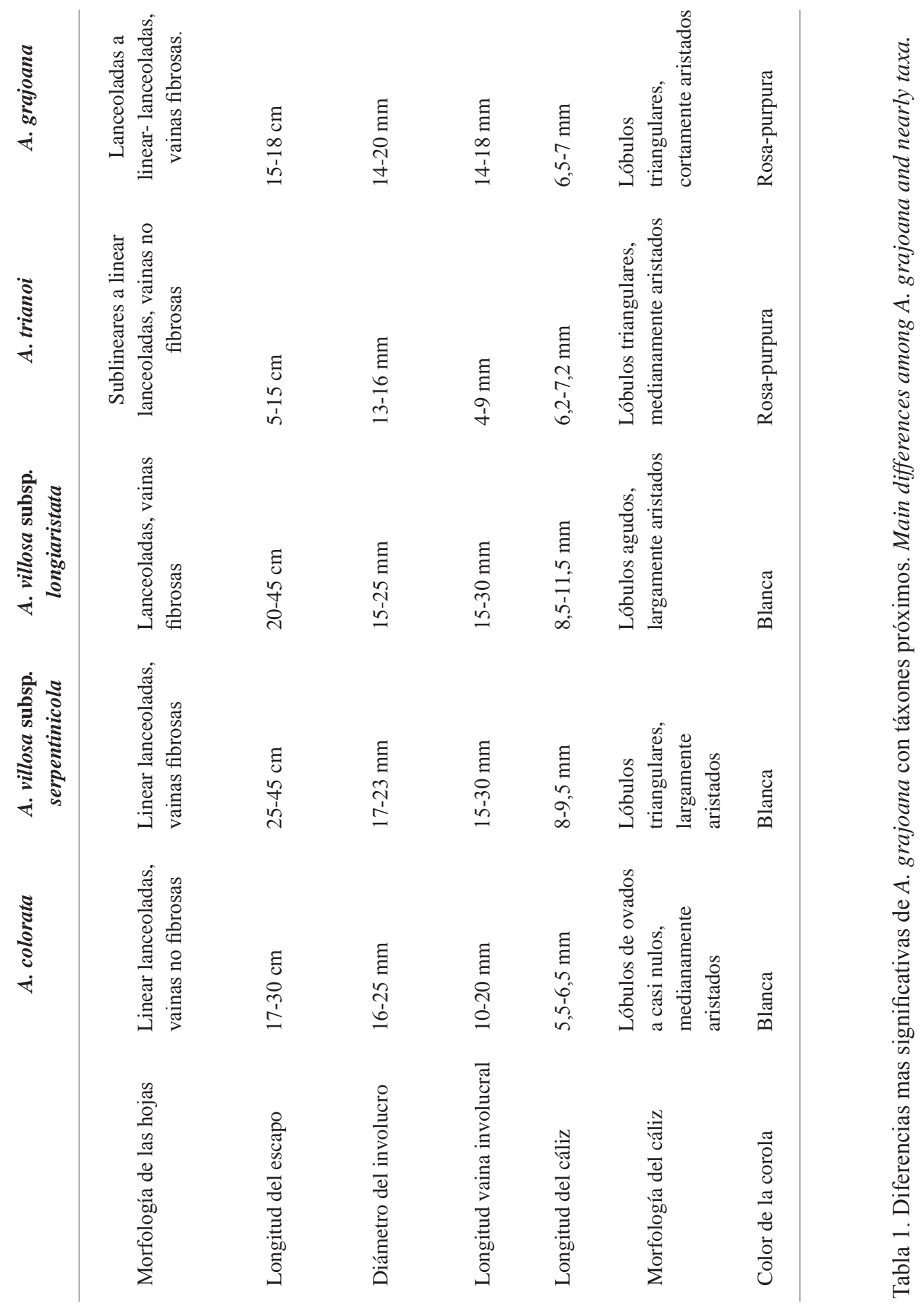


(1987) (En Peligro (B2ab(ii,iv)) se refieren exclusivamente a las poblaciones de $A$. villosa subsp. serpentinicola sobre peridotitas.

\section{Otro material estudiado}

Armeria bourgaei Boiss. ex Merino subsp. bourgaei Jaén: Albanches de Úbeda. Sierra de Mágina. Sobre la Fuente del Espino. VG5976. calizas. 1800 m.s.m. 18/06/2002. Cabezudo, Pérez Latorre \& Gil (MGC 52665, 53010); Almería: María. Sierra de María. Cara Norte, sobre los Alaminos. 1250-1750 m.s.m. 07/06/1995. Cabezudo, Pérez Latorre, Blanca \& Cueto (MGC 39693)

Armeria bourgaei subsp. Ianceobracteata (Lawrence) Nieto Feliner

Granada: Sierra de Guillimona. WH30. Pastos sobre calizas. 1750 m.s.m. 14/06/1986. Bayón, Galán \& Nieto Feliner. (MA386477); Sierra del Cuarto. Lieux arides, sur le calcaire. 1800m.s.m. 06/1902. (MA145769)

Armeria bourgaei subsp. willkommiana (Bernis) Nieto Feliner

Murcia: Benizar. Rincón de las Cuevas. WH8835. Roquedo, calizas. 1000 m.s.m. 24/04/1997. C, Aedo, I. Álvarez, S. Castroviejo, R. Morales, F. Muñoz, M. Velayos \& Al. (MA591195); Sierra de Espuña, Morrón de Espuña. In rupestribus calcareis. 23/06/1947. C. Vicioso (MA386529).

\section{Armeria colorata Pau}

Málaga: Istán. Entre el puerto del Algarrobo y el de la Refriega. UF1938055998. Matorrales y taludes. Peridotitas.916-1166 m.s.m. 15/06/2012. Cabezudo \& Casimiro-Soriguer Solanas (MGC75674); ibídem. Sierra Real. Serpentinas. 21/06/1996. Navas, Gil \& Navas (MGC44201); ibídem. Cerro del Duque UF2055. Serpentinas. 940 m.s.m. 7/06/1996. Gil, Pérez Latorre \& Navas (MGC 44198); ibídem. UF2056. Zona rocosa. Serpentinas. 26/07/2001. Navas, Gil \& Navas. (MGC 49301). Casares. Sierra Bermeja. Pico de los Reales. Serpentinas. Grandes bloques de piedra. 11/06/1996. Navas, Cabezudo \& Navas (MGC 44197, 44199). Tolox: Sierra de Tolox. Cerro Corona. Peridotitas. 30/6/1996. Gil, Pérez Latorre \& Navas ( MGC 47722). Coín. Sierra
Alpujata. Rio Pereila. UF 3952. Peridotitas. 680 m. s. m. 13/5/2009. Cabezudo, Pérez Latorre \& CasimiroSoriguer Solanas (MGC 69810).

Armeria filicaulis (Boiss.) Boiss. subsp. filicaulis Granada: Alhama de Granada. P.N. Sierra de Tejeda, Almijara y Alhama. Subida al Lucero. VF 1981. Dolomías. 1330-1510 m.s.m. 17/06/2004. Cabezudo, Pérez Latorre, Caballero \& Gavira. (MGC 59672). Málaga: Cómpeta. Sierra Almijara. Del Puerto del Collado a Cerro Canillas. VF 1481. Dolomías. 1000 m.s.m. 2/07/1996. Gil, Cabezudo \& Pérez Latorre (MGC 42240)

Armeria filicaulis subsp. nevadensis Nieto Feliner, Roselló \& Fuertes

Granada: Sierra Nevada. El Dornajo. 4/08/1984. Cabezudo, Guerra \& Pérez. (MGC 41679). Monachil. Sierra Nevada. Collado del Diablo. VG6407. Enebral-piornal. Micaesquistos. 2300m.s.m. 27/07/2010. Navarro, Rodriguez \& Casimiro-Soriguer Solanas (MGC72167)

Armeria malacitana Nieto Feliner

Málaga: Alhaurín el Grande. Sierra de Mijas. Puerto de la Encina. UF 55. 27/05/1983. Cabezudo \& Pérez Sanz (MGC 15521); ibídem. 19/06/1991. Cabezudo \& Hidalgo (MGC 32222); ibídem. Pico Mijas. UF5153. Arenas dolomíticas. 1150 m.s.m. 24/05/1994. Gil, Navas \& Navas (MGC 39569); ibídem. Subida al repetidor. Ternero \& Gil. (MGC44761). Ojén. Puerto de Ojén. Dolomías sacaroideas. 12/05/1988. Cabezudo, Nieto \& Pérez Latorre. (MGC24589). Coín. Sierra Blanca. La Albuqueira. UF 4055. Dolomías. 13/05/2009. Cabezudo, Pérez Latorre \& Casimiro-Soriguer Solanas. (MGC 69814)

\section{Armeria trianoi Nieto Feliner}

Córdoba: Priego. La Tiñosa. Zona cacuminal. UG 9038. Roquedos umbríos. Calizo-dolomías. 1550 m.s.m. 15/06/1998. Triano, Jiménez Conejo et al. (MA 631755); Sierra Horconera. Pico Bermejo, bajando al canalizo de Peñalisa. UG54. 20/05/1982. G. Fernández. J. García \& S. Silvestre (SEV76396, $82315,82316,82314$ )

Armeria villosa Girard subsp. villosa Málaga: Yunquera. Sierra de las Nieves. 
Entre la Cañada de los Hornillos y el Peñón de los Enamorados. UF26. 1350-1750 m.s.m. Cabezudo, Pérez Latorre, Gil, Navas \& Navas (MGC 46786); Ibídem. Puerto de los Hornillos. UF2366. Calizas. 1300 m.s.m. 12/06/2002. Navas, Gil, Gavira \& Navas (MGC52284); Ibídem. Cañada de la Encina. UF2266. Matorral. Calizas. 1200m.s.m. 15/06/2003. Navas \& Trias (MGC55178). Ronda. Sierra de las Nieves. Cañada de las Ánimas. UF2065. Tomillar nitrófilo. Calizas. 1360 m.s.m. 17/06/1997. Pérez Latorre, Navas, Gil \& Navas (MGC46063); Ibídem. Subida al puerto de los Pilones por la Pista forestal. UF1962. Calizas. Matorral. 06/06/2001. Cabezudo et al. (MGC 49352). Tolox. Sierra de las Nieves. Picacho de Fatalandar. UF 2562. Rupícola. Dolomías. 2/7/214. Cabezudo, Pérez Latorre \& Casimiro-Soriguer Solanas. (MGC 79-626).

Armeria villosa subsp. alcaracensis Nieto Feliner Albacete: La Mesta. 2/06/1934. González Albo (MA349602); Bienservida. Confluencia del río Guadalmena y cañada del Conejo. WH2367. Pastizales sobre cuarcitas. 13/05/1996. J. Herranz. (MA586744); Vianos. WH4177. Cuarcitas y margas triásicas. 960 m.s.m. 28/05/1996. J. Herranz (MA586743)

Armeria villosa subsp. bernisii Nieto Feliner Granada: Alhama de Granada. Sierra Almijara. Carril de la Resinera al Navachica. VF2781. Dolomías. 11/07/1996. Cabezudo, Pérez Latorre, Gil, Navas \& Navas (MGC 44202); Ibídem. Cortijo de Pantaneta, bajo Loma del Águila. VF2582. 1100m.s.m. 2/06/2004. Cabezudo, Pérez Latorre, Caballero \& Gavira (MGC59678). Órgiva. Sierra de Lujar. Cara NE. Calizas. 1660m.s.m. 17/06/1996. Pérez Latorre, Navas \& Navas (MGC44194). Mecina Bombarón. Pista al Pto. de la Ragua. 1600-1800 m.s.m. 19/07/1995. Cabezudo, Talavera \& Navas (MGC 42640). Ótivar. Sierra Almijara. Navachica, cara Oeste. VF 2976. Dolomías. 11/07/1996. Cabezudo, Pérez Latorre, Gil, Navas \& Navas MGC (44193); Almería: María. Sierra de María, cara N. sobre los Alaminos. 1270-1750 m.s.m. 07/06/1995. Cabezudo, Pérez Latorre, Blanca \& Cueto (MGC39691)

Armeria villosa subsp. longiaristata (Boiss. \& Reut.) Nieto Feliner

Málaga: Alfarnate. Sierra de Alfarnate.
26/06/1919. Gros. (MA 145441). Antequera. Sierra Llana. In glareosis. 14/06/1930. Vicioso. (MA 145419). El Burgo. Sierra Cabrilla. UF3172. Rupícola. Calizas. 26/05/2010. Gavira \& CasimiroSoriguer Solanas (MGC71692); ibídem. Sierra de las Cabras. Collado de los Lastonares. UF6992. Arcillascalizas. 1192m.s.m. 09/07//2012. García-Sánchez \& Casimiro-Soriguer Solanas (MGC75791); Ibídem. Sierra de Huma. Zona superior. UF4388. Rupícola. Calizas. 1050 m.s.m. 30/05/2013. Cabezudo, Pérez Latorre, Hidalgo \& Casimiro-Soriguer Solanas (MGC77619). Parauta. Cancha Almola. UF0860. Dolomías. 1100m.s.m. 12/06/2004. Gavira (MGC60717). Valle de Abdalajis. Sierra de Huma. Paredón. Calizas. 840m.s.m. 14/06/1996. Cabezudo \& Pérez Latorre (MGC44200). Ronda. Sierra de las Nieves. Subida al Pto. De los Pilones. Calizas. 12/06/1996. Nieto \& Navas (MGC43321). Granada: Puerto de los Alazores. 09/06/1965. Rivas Goday \& Borja (MGC4361). Jayena. Sierra Almijara. Bco. de la casa de Orihuela. VF3184. Dolomías. 1250 m.s.m. 24/06/2003. Cabezudo, Pérez Latorre, Caballero \& Navas (MGC60339). Cádiz: Grazalema. Sierra del Pinar. Subida al Torreón. TF8371. 1000-1600 m.s.m. 17/07/1984. Aparicio \& Silvestre (MGC29522)

Armeria villosa subsp. provillosa (Bernis) Nieto Feliner

Ciudad Real: Montes alrededor de la Vega del río Tovar. 25/05/1934. González Albo (MGC 145496). Aldea de San Pedro. Lagunas de Ruidera. 28/05/1933. González Albo (MA145502). Jaén: Villarodrigo. La Mangada. WH2756. Prados sobre margas calizas. 980m.s.m. 19/06/1984. Fernández (MA403427)

DEDICATORIA. Dedicamos este trabajo a Carlos Vicioso, Francisco Bernis, Gonzalo Nieto Feliner y Juan A. Devesa. Carlos Vicioso recolectó y estudió por primera vez la Sierra de Alcaparaín y suyo es el pliego original (Holotypus) de A. grajoana (sub. A. villosa var. carratracensis). A Francisco Bernis y Gonzalo Nieto Feliner por su larga y fructífera dedicación al estudio del complejo género Armeria. Agradecemos a Juan Antonio Devesa la lectura crítica del manuscrito y sintiendo no haber tenido en consideración alguna de sus sugerencias sobre nomenclatura. 


\section{BIBLIOGRAFÍA}

BERNIS, F. -1955- Revisión del género Armeria con especial referencia a los grupos ibéricos. Parte $2^{\mathrm{a}}$. Anales Inst. Bot. Cavanilles 12 (2): 77-252

BLANCA, G., B. CABEZUDO, M. CUETO, C. FERNÁNDEZ LÓPEZ \& C. MORALES TORRES. ( Eds.) -2009- Flora Vascular de Andalucía Oriental. Junta de Andalucía . Sevilla. CABEZUDO, B., Y. GIL, A. V. PÉREZ LATORRE, P. NAVAS, D. NAVAS \& T. NAVARRO -2000Armeria villosa subsp. carratracensis ( Bernis) Nieto Feliner. In Blanca, G., B. Cabezudo. J.E. Hernandez-Bermejo, C. M. Herrera. J. Muñoz \& B. Valdés (Eds.). Libro rojo de la flora silvestre amenazada de Andalucía. Tomo II, Especies Vulnerables. Junta de Andalucía.

CABEZUDO, B., Y. GIL, A. V. PÉREZ LATORRE, P. NAVAS \& D. NAVAS -2003- Armeria villosa subsp. carratracensis ( Bernis) Nieto Feliner. In Bañares, Á., G. Blanca, J. Guemes, J. C. Moreno \& S. Ortiz (Eds.). Atlas y Libro Rojo de la Flora Vascular, Amenazada de España. DGCN. Madrid.

CEBALLOS, L. \& C. VICIOSO -1933- Estudio sobre la vegetación y la flora forestal de la provincia de Málaga. Instituto Forestal de Investigaciones y Experiencias. La Moncloa, Madrid.

DEVESA, J.A. \& R. PINTO DA SILVA -1984Armeria maritima subsp. alpina (Willd.) Pinto da Silva en el sur de España. Anales del Jardín Botánico de Madrid 40(2): 466-467.

LÓPEZ GONZÁLEZ, G. -1975- Contribución al estudio florístico y fitosociológico de Sierra de Aguas. Acta Bot. Malacitana 1: 81-205.

NIETO FELINER, G. -1987- El género Armeria (Plumbaginaceae) en la Península Ibérica: aclaraciones y novedades para una síntesis. Anales Jardín Botánico de Madrid, 44(2): 319-348
NIETO FELINER, G. -1990- Armeria Willd. In: Castroviejo S., Lainz M., López González G., Montserrat P., Muñoz Garmendia F., Paiva J., Villar L., eds. Flora Iberica. Madrid: Real Jardín Botánico, vol. 2, 642-721.

NIETO FELINER, A. IZUZQUIZA \& A.R. LANSAC -1996- Natural and experimental hybridization in Armeria (Plumbaginaceae): A. villosa subsp. carratracensis. Pl. Syst. Evol. 201: 163-177.

NIETO FELINER, G., J. FUERTES AGUILAR \& J. A. ROSSELLÓ -2001-A new species of Armeria (Plunbaginaceae) from southern Spain with molecular and morphometric evidence on its origin. Bot. J. of the Linnean Society 135:71-84 PÉREZ LATORRE, A. V. \& B. CABEZUDO -2002La flora y el paisaje vegetal de la provincia de Málaga: importancia y conservación. Jábega 90: 25-39.

PÉREZ-LATORRE, A. V., N. HIDALGO \& B. CABEZUDO -2013-Composition, ecology and conservation of sout-Iberian serpentine flora in the context of the Mediterranean basin. Anales Jardín Botanico Madrid 70:62-71.

RIVAS MARTÍNEZ, S. -1988- Bioclimatología, biogeografía y series de vegetación de Andalucía Occidental. Lagascalia 15 (extra): 91-119.

RIVAS MARTÍNEZ, S., A. ASENSI, J. MOLERO MESA \& F. VALLE -1991- Endemismos vasculares de Andalucía. Rivasgodaya 6: 5-76

RIVAS -MARTINEZ, S. \& coautores -2011aMapa de series, geoseriesy geopermaseries de vegetación de España. Itinera geobotanica 18-1: 5.424 .

RIVAS -MARTINEZ, S. \& coautores -2011bMapa de series, geoseriesy geopermaseries de vegetación de España. Itinera geobotanica 18-II: 425-800.

VALDÉS, B., S. TALAVERA \& E. F. GALIANO -1987- Flora Vascular de Andalucía Occidental. Ed. Ketres. 